\title{
Impact of an at home exercise program in the pediatric, adolescent, and young adult cancer population newly diagnosed with acute lymphoblastic leukemia
}

Punit Vyas ${ }^{1}$, Danielle Halsey ${ }^{2}$, Nicholas Kelly ${ }^{3}$, Melissa Sherman ${ }^{3}$, Brooklyn Herbert ${ }^{4}$, Khadijeh

Bijangi-Vishehsaraei ${ }^{1,2}$, Riggs Klika ${ }^{4}$, NiCole Keith ${ }^{3}$, Jamie Renbarger ${ }^{1,2}$, Tammy Sajdyk ${ }^{1,2}$ ${ }^{1}$ Indiana University School of Medicine; ${ }^{2}$ Indiana University School of Medicine, Department of Pediatrics; ${ }^{3}$ Indiana University Purdue University, School of Health and Human Sciences;

${ }^{4}$ University of Indianapolis

Background: Over 30,000 cases of leukemia are diagnosed each year in the United States. For individuals ages $0-39$, the one-year relative survival rate is $84.5 \%$, and the 5 -year survival is $94 \%$. Improved survival rates come with long-term health challenges due to treatment, including cardiovascular disease, obesity, diabetes, fatigue, low quality of life (QOL), and reduced physical activity (PA). Current literature shows structured exercise in adult survivors can be beneficial for improving the long-term health effects resulting from treatment. Exercise induces the release of many growth factors into the bloodstream that aid in repair of the body following insult. Three factors specific to nerve damage is brain-derived neurotrophic factor (BDNF), nerve growth factor (NGF), and Platelet-derived growth factor (PDGF). The benefits of exercise seen in adult survivors may translate to young survivors. We hypothesized that an 8-week at home exercise program in newly diagnosed acute lymphoblastic leukemia (ALL) patients (ages 5-25) would improve outcomes compared to baseline or a control group. The preliminary findings presented are based on the initial ten subjects and include physical, biomarker and self-reported outcomes.

Experimental Methods: Newly diagnosed ALL patients were assigned to an 8-week at home exercise intervention during the consolidation phase of treatment. Fitbit activity tracker data and questionnaires were used for assessing adherence and feasibility. Blood samples were taken at baseline and at week eight.

Results: Subjects were not able to complete over $75 \%$ of the exercise intervention; however, they did increase their overall physical activity and their neuropathy scores decreased compared to baseline. BDNF significantly increased in the control group, but not in the exercise group.

Conclusion: The results indicate that an exercise program in this population during the consolidation phase may not be feasible. It also supports the association between neuropathy, physical activity, and BDNF levels after long term exercise. 